\title{
Psicoterapia Interpessoal no Tratamento da Perturbação Depressiva Maior
}

Catarina Oliveira Lucas $\star$

\begin{abstract}
Resumo
A terapia interpessoal é um modelo de terapia breve formulado para o tratamento da perturbação depressiva maior, tendo como precursores Gerald Klerman e Mirna Weissman. Esta visa o alivio dos sintomas associados à depressão abordando os problemas interpessoais que os originaram e perpetuam. Esta modalidade de terapia refere as perturbações como resultantes da existência de problemas numa das quatro áreas interpessoais principais: luto, transição de papéis, disputas de papéis interpessoais e défices interpessoais. Deste modo, a terapia incidirá na área afectada. Esta demonstra bons indicadores de eficácia e apesar de formulada para o tratamento da depressão, a terapia interpessoal tem sido adaptada e expandida a outras perturbações.
\end{abstract}

Palavras-chave: terapia interpessoal; depressão; disputas e défices interpessoais; transição de papéis.

\section{Interpersonal Psychotherapy in the Treatment of Major Depressive Disorder}

\begin{abstract}
Interpersonal therapy is a brief therapy model formulated for the treatment of major depressive disorder having as precursors Gerald Klerman and Mirna Weissman. It aims to relieve symptoms associated with depression, referring the interpersonal problems that originate and perpetuate the depression. This form of therapy refers the disorders as resulting from problems in one of the four main interpersonal areas: grief, role transitions, role disputes interpersonal and interpersonal deficits. Thus, therapy will focus on the affected area. This therapy demonstrates good indicators of efficiency and although formulated for the treatment of depression, interpersonal therapy have been adapted and expanded to other disorders.
\end{abstract}

Keywords: interpersonal therapy; depression; disputes and interpersonal deficits; role transition.

\footnotetext{
^Psicóloga. Mestrado em Psicologia Clínica e da Saúde pela Universidade da Beira Interior (Covilhã). Morada: Avenida Pedro Álvares Cabral. 6000-085 Castelo Branco, Portugal.

E-mail: catarina.lucas@live.com.pt
} 


\section{Fundamentos da Terapia InTERPessoal}

A terapia interpessoal teve a sua origem em 1970, surgindo como uma forma de terapia breve para o tratamento da perturbação depressiva maior, tendo como precursores Gerald Klerman e Mirna Weissman. Esta baseava-se nas ideias da escola interpessoal de psicanálise de Sullivan, nas contribuições de Adolf Meyer, nos estudos sobre o luto de Freud e na teoria do apego de Bowlby (SCHESTATSKY; FLECK, 1999; CURRY, 2001), sendo os seus princípios publicados sobre a forma de manual (FLECK, 2008).

Assim, esta tem por base teorias e estudos empíricos que relacionam o stress, os acontecimentos de vida e o comprometimento social com a depressão (MUFSON et al., 2004), estando simultaneamente associada à compreensão psicodinâmica, à utilização de técnicas cognitivo-comportamentais e ao envolvimento da família (ROSSELO; BERNAL, 1999). Tem em comum com as abordagens dinâmicas a preocupação com a vida passada do sujeito e o reconhecimento da importância das experiencias de infância, todavia, as primeiras focam-se no intrapsíquico, enquanto que a terapia interpessoal se foca nas relações interpessoais (SCHWARTZ, 2001).

Foi inicialmente formulada para ser realizada ao longo de doze sessões, tendo como objetivo o alívio dos sintomas, sendo que para tal aborda os problemas interpessoais que os originaram e perpectuam. Assim, mediante esta forma de terapia, as perturbações psiquiátricas surgem sempre num contexto interpessoal (MELLO, 2004). Além disto, a terapia interpessoal não faz qualquer suposição sobre a etiologia da depressão, acreditando que o seu desenvolvimento ocorre num contexto social e interpessoal, sendo o tratamento influenciado pelas relações interpessoais (SCHESTATSKY; FLECK, 1999; MUFSON et al., 2004). Atribui ainda especial ênfase à formação dos sintomas e à disfunção social associada à depressão, ao invés de abordar os aspectos ligados à personalidade (MELLO et al., 2005). Foca-se na relação entre o início da sintomatologia e os problemas interpessoais actuais, tendo subjacente o conceito de doença ou patologia. Esta terapia encara as perturbações como resultantes de problemas numa das quatro principais áreas interpessoais, nomeadamente o luto (perda de uma pessoa significativa), a transição de papéis (dificuldade para gerir as mudanças que ocorrem ao longo da vida), as disputas de papéis interpessoais (conflito numa relação) e os défices interpessoais (dificuldade no relacionamento com os outros). A terapia incidirá assim na área afectada (SCHESTATSKY; FLECK, 1999).

Nesta perspectiva, a terapia interpessoal compreende a depressão como possuindo três componentes: a formação de sintomas (início do humor depressivo, dos sinais e dos sintomas), funcionamento social (impacto da depressão no envolvimento social e relações pessoais) e personalidade (traços de personalidade que predispõem a pessoa à depressão) (MUFSON et al., 2004). No entanto, esta centra-se apenas nos dois primeiros, já que a terapia interpessoal é um tratamento breve, e como tal não conseguirá alcançar mudanças a nível da personalidade num curto período de tempo (FLECK, 2008). 
Esta forma de terapia foi revista por Moreau e colaboradores em 1991, para assim poder ser utilizada com adolescentes. Esta adaptação envolveria questões como o afastamento dos pais, problemas com a autoridade, perdas de pessoas significativas, pressões do grupo de pares, entre outros (BAHLS, S.; BAHLS, F., 2003).

Assim, a terapia interpessoal deve ser realizada por profissionais treinados na área da saúde mental, podendo ser, ou não, utilizada conjuntamente com terapia farmacológica (WEISSMAN, 2005).

\section{INDICAÇÕES TERAPÊUTICAS}

A terapia interpessoal é de fácil aplicação, possuindo resultados muito positivos no tratamento da depressão (MELLO, 2004). Ao longo dos anos, a sua aplicação tem sido alargada e adaptada a outras perturbações psiquiátricas (WEISSMAN 2000 apud MELLO et al., 2005), tais como perturbações alimentares, perturbação de ansiedade (FRANK et al., 2000) e depressão em populações específicas como por exemplo idosos e adolescentes (MILLER, 2008).

Além disto, estudos recentes demonstram que a terapia interpessoal possui um efeito benéfico também no tratamento da perturbação de stress pós traumático (CAMPANINI et al., 2010), bem como na perturbação de personalidade borderline (BELLINO; RINALDI; BOGETTO, 2010). No caso concreto do tratamento da bulimia nervosa, a terapia interpessoal surge como segunda opção, quando a terapia cognitivo-comportamental não surte os efeitos desejados, demonstrando resultados bastante positivos (ARCELUS et al., 2009). Esta pode ainda ser útil nos casos de esquizofrenia onde a depressão se encontra presente (LACAZ; BRESSAN; MELLO, 2005).

\section{Estratégias de INTERVEnÇÃo Na Terapia InTERPESSOAL}

Habitualmente, a terapia interpessoal é realizada através de três fases, sendo que a primeira (fase inicial) tem a duração de uma a três sessões, onde o terapeuta obtém a história clínica, identifica a área interpessoal que possui o problema e realiza a psicoeducação. A segunda fase (fase intermediária) é realizada da quarta à oitava sessão, sendo trabalhada a área afectada através de técnicas específicas. A fase final possui também a duração de uma a três sessões e é nesta fase que é fornecido suporte ao paciente, incentivada a independência, reconhecimento e consolidação dos ganhos e prevenção de recaídas (BAHLS, S.; BAHLS, F., 2003; MELLO, 2004).

Deste modo, é na segunda fase que as áreas identificadas como problemáticas são trabalhadas, sendo que, no que concerne ao luto, a intervenção visa a facilitação do processo de luto e ajudar o paciente a reencontrar interesses e relações que substituam a relação que se perdeu. Para tal, utilizam-se como estratégias a análise dos sintomas depressivos, relacionando o inicio dos sintomas com a morte da pessoa significativa. É feita a reconstrução da relação com a pessoa falecida e analisada a sequência e consequências dos acontecimentos, antes e depois da morte da pessoa significativa. Além disto, é feita a descrição de sentimentos asso- 
ciados, considerando-se possíveis formas de o paciente voltar a relacionar-se com os outros. O terapeuta irá trabalhar as relações entre o paciente e a pessoa falecida, sendo que o trabalho terapêutico busca encontrar a relação real entre ambos, trabalhando os conflitos relacionados a esta. É assim trabalhada a perda, abrindo caminho para relações actuais que o ajudem a superar a relação imaginária com a pessoa perdida (LEVENSON et al., 2002).

Relativamente às disputas interpessoais (ex: conflitos conjugais), os objetivos são identificar as disputas, escolher o plano de acção, mudar expectativas e falta de comunicação e trazer uma resolução satisfatória. Assim, são analisados os sintomas depressivos, verificam-se as relações de disputa com outros significativos e determina-se o estádio da disputa (renegociação, impasse ou dissolução). Dependendo do estádio, a estratégia difere, sendo que o objectivo é ajudar o paciente a reconhecer os seus sentimentos e encontrar estratégias para controlá-los. A intervenção na área da transição de papéis tenta ajudar o paciente a aceitar a perda do seu antigo estatuto, a olhar o novo papel mais positivamente e a restaurar a auto-estima. Para tal, o terapeuta relaciona os sintomas depressivos com as actuais dificuldades a nível das estratégias para lidar com as recentes mudanças de vida. Analisam-se os aspectos positivos e negativos do antigo e actual papel e são explorados os sentimentos em relação ao que foi perdido e as oportunidades que podem surgir neste novo estatuto. O paciente é encorajado a desenvolver um sistema de suporte social e a adquirir novas estratégias necessárias no seu novo estatuto. $\mathrm{O}$ diagnóstico da dificuldade nesta área ocorre quando a pessoa apresenta dificuldades em lidar com mudanças de vida. Deste modo, procuram-se formas de enfrentar o novo papel e de lidar com as dificuldades, reformulando a forma de agir. Por fim, na área dos défices interpessoais, o terapeuta pretende reduzir o isolamento social e encorajar a criação de novas relações. Tal como nas outras áreas são analisados os sintomas depressivos e relacionados com os problemas de isolamento social. Analisam-se as relações significativas passadas, incluindo aspectos positivos e negativos e são explorados os padrões relacionais que mais se repetem. Além disto discute-se com o paciente os sentimentos negativos e positivos em relação ao terapeuta e outros relacionamentos paralelos (LEVENSON et al., 2002).

Muitas das técnicas utilizadas nesta terapia são comuns à terapia psicodinâmica, passando estas por técnicas de carácter exploratório, subdividindo-se assim nas técnicas de exploração não directiva e técnicas de levantamento directivo de material. Assim, as primeiras são realizadas através de questões abertas e gerais, podendo utilizar-se o reconhecimento suportivo (técnica não directiva que consiste em dizer "hum-hum", "entendo", entre outros) e o silêncio receptivo (o terapeuta mostra interesse e encoraja o paciente a falar). No que concerne às técnicas de levantamento directivo de material, são utilizadas quando o terapeuta faz um questionamento sobre determinado tópico. Além destas, o terapeuta recorre ao encorajamento da expressão dos afectos por parte do paciente, que permite a aceitação de afectos negativos sobre aspectos que não podem ser alterados e o desenvolvimento de novos afectos que permitam a mudança. Outra técnica utilizada é a clarificação, através da qual o que é dito pelo paciente é reestruturado a fim de que este tome consciência do mesmo. Citam-se ainda a análise da comunicação 
(que tem como objectivo identificar as falhas comunicacionais do paciente, para assim o ajudar a desenvolver habilidades de comunicação), modificação comportamental (através da educação e modelagem), a análise das decisões (o terapeuta incentiva o paciente a considerar várias soluções) e o role-playing (o terapeuta faz o papel de alguém significativo para o paciente, explorando os sentimentos e estilos de comunicação, a fim de criar formas de comunicação mais adaptativas). A educação acerca da patologia é um aspecto manifesto também na terapia interpessoal, sendo que esta é feita através do diálogo sobre o diagnóstico, sintomas e tratamento (MELLO, 2004; FLECK, 2008).

\section{Terapia InTERPessoal aplicada aO Tratamento da PERTURbaÇão DEPRESSIVA MAIOR}

A psicoterapia interpessoal faz parte das terapias aplicadas na perturbação depressiva maior, mais testadas nos últimos anos (SCHESTATSKY; FLECK, 1999), e Swartz et al. (2004), através do seu estudo comprovaram a sua eficácia. Esta deve ser realizada ao longo de 12/16 sessões, explorando a relação entre os sintomas ligados ao humor e as relações interpessoais. O terapeuta reconhece a importância de factores inconscientes mas a sua intervenção dirige-se apenas a fenómenos conscientes e pré conscientes (FLECK, 2008).

Mediante a terapia interpessoal, as dificuldades nas relações interpessoais estão presentes não só durante a depressão, mas também antes dela se iniciar. No entanto, esta não pretende descodificar etiologia desta perturbação, mas sim verificar a relação entre os problemas interpessoais e o inicio e manutenção da depressão, propondo um tratamento com base nisto (SCHESTATSKY; FLECK, 1999). Deste modo, o contexto social e interpessoal do desenvolvimento da depressão pode ajudar a descobrir a origem dos sintomas, sendo que o desenvolvimento de novas estratégias sociais está na base do tratamento da depressão actual, reduzindo também a vulnerabilidade à recaída (WEISSMAN; MARKOWITZ; KLERMAN, 2007). É importante conhecer a influência dos factores interpessoais no desencadeamento e manutenção da depressão, bem como compreender a influência da depressão nas relações interpessoais (FLECK, 2008).

Relativamente ao tratamento da depressão, a terapia interpessoal tem como objectivos a redução dos sintomas e o incremento da qualidade das relações interpessoais. Estes objectivos são alcançados através de sessões semanais face-a-face, nas quais a depressão é explicitamente diagnosticada, o paciente é educado sobre a depressão e suas causas, bem como sobre os vários tratamentos disponíveis. É também identificado o contexto social da depressão e demonstrado ao paciente que este deve ter um papel activo na implementação de estratégias (KLERMAN; WEISSMAN, 1993). Inicialmente, o programa terapêutico é simples e pragmático, estando organizado de forma a ajudar o paciente a compreender a situação em que se encontra (MARKOWITZ, 1998) e empregando o modelo médico de doença mental, atribuindo assim ao sujeito um estatuto de “doente" (MARKOWITZ; SWARTZ, 2007). 
Deste modo, a terapia interpessoal no tratamento da perturbação depressiva maior envolve três fases, sendo que na fase inicial, as primeiras sessões se destinam à identificação das áreas problemáticas e à realização do diagnóstico. Seguidamente o terapeuta deve facultar ao paciente a informação necessária sobre a patologia e o tratamento. Deve também direccionar a atenção do paciente para o início dos sintomas e para os motivos que o levaram a procurar ajuda. Além disto, deve ser feita uma análise das relações interpessoais que envolva a exploração do tipo de interacção com as pessoas, das expectativas relativas à relação, dos aspectos positivos e negativos da relação e das mudanças desejadas na relação (BAHLS, S.; ITO, 2003).

Relativamente à fase intermediária, o terapeuta deve centrar-se nas áreas definidas como problemáticas na primeira fase, ajudando o paciente a identificar as pessoas com quem tem problemas no relacionamento, quais são esses problemas e formas de melhorar esses relacionamentos. A terapia deve centrar-se no máximo em duas áreas problemáticas, sendo que estas podem alterar-se ao longo da mesma. Os objectivos desta fase passam por ajudar o paciente a expressar os aspectos relativos à área-problema identificada, analisar o estado emocional do paciente e prevenir o abandono do tratamento. As quatro áreas-problema levadas em conta por esta terapia podem estar interligadas, sedo que o terapeuta deve identificar aquela que será o alvo da terapia, de forma a centrar a sua intervenção no problema que afecta as relações interpessoais do sujeito. O paciente deve ainda alterar as suas expectativas negativas face às relações ou aceitar aquelas que não possuem solução (MARKOWITZ, 1998).

Por fim, na última fase da terapia, deve ser discutido o final da terapia, pois com o seu término o paciente possui a tarefa de fechar uma relação e consolidar as suas competências para lidar com futuros problemas sem a ajuda do terapeuta, sendo que quando tal não ocorre, os sintomas depressivos podem regressar, já que o paciente pode pensar que sem o apoio do terapeuta não conseguirá resolver os problemas. Posto isto, é necessário ensinar ao paciente quando é que este o deve procurar novamente. Quando a terapia não surtiu qualquer efeito, outras modalidades de intervenção devem ser consideradas (BAHLS, S.; ITO, 2003).

Importa ainda referir a terapia interpessoal de manutenção, que foi desenvolvida a partir do pressuposto que o paciente possui um contexto interpessoal que pode desencadear a recaída. Assim, esta visa a manutenção da recuperação e a redução da vulnerabilidade a episódios futuros e enquanto que a terapia interpessoal se foca na mudança do contexto, a terapia interpessoal de manutenção visa o reforço desse mesmo contexto, ajudando o paciente na resolução dos problemas. As sessões nesta modalidade da terapia interpessoal são realizadas uma vez por mês (ELKIN, 1989 apud SCHESTATSKY; FLECK, 1999).

\section{CONSIDERAÇõES FINAIS}

A terapia interpessoal é um modelo de intervenção breve, formulada especificamente para a intervenção na perturbação depressiva maior, tendo recebido a influência das terapias dinâmicas. No entanto, esta valoriza os relacionamen- 
tos interpessoais em detrimento dos processos inconscientes. Mediante a terapia interpessoal, são as relações interpessoais que desencadeiam e perpetuam a depressão, todavia não emite qualquer formulação sobre a etiologia da mesma, intervindo em quatro áreas-problema (luto, transição de papéis, disputa de papeis e défices interpessoais) e tendo como objectivo a melhoria da sintomatologia depressiva. Depois da sua aplicação no tratamento desta patologia, a sua extensão a outras perturbações tem vindo a ser amplamente estudada e a sua eficácia comprovada através de diversos estudos científicos.

É irreal e ilusório pensar que uma terapia pode tratar todas as vertentes de uma patologia, no entanto a terapia interpessoal oferece uma visão bastante ampla do sujeito e do meio que o rodeia, actuando nas diversas dificuldades dos sujeito, que condicionam o seu funcionamento social. Portanto esta assume-se como uma terapia bastante promissora no tratamento de algumas patologias. No entanto, aspectos como crenças disfuncionais parecem ser insuficientemente trabalhadas, sendo este um aspecto vulgarmente presente nos sujeitos deprimidos ou portadores de outra perturbação.

Apesar disto, a terapia interpessoal apresenta-se como uma eficaz alternativa quando outras modalidades terapêuticas não resultam, devendo os seus recursos ser aproveitados em prol da melhoria da sintomatologia do paciente. Concretamente, esta surge vulgarmente como segunda opção no tratamento de perturbações alimentares, sendo considerada quando outras terapias, nomeadamente a terapia cognitivo-comportamenteal não surte efeito. Além disto, por ser uma terapia breve, questões económicas se colocam relativamente ao paciente, pois esta pode ser menos dispendiosa em termos monetários e temporais, comparativamente a outras modalidades terapêuticas de durabilidade maior, tal como a psicanálise.

Vários são os estudos que demonstram a sua superior eficácia comparativamente à intervenção farmacológica, existindo igualmente estudos que demonstram a mesma eficácia entre a terapia interpessoal e outras modalidades terapêuticas igualmente estudadas.

Constitui também uma vantagem desta modalidade terapêutica a sua fácil aplicação e a boa adesão dos sujeitos ao tratamento, devendo a sua implementação ser sempre considerada aquando da escolha do tratamento. No entanto, esta terapia apenas se revela de fácil aplicação para técnicos especializados em saúde mental, não devendo nunca ser colocada em prática por pessoas sem a devida formação e treino. Estes técnicos são habitualmente os psicólogos, já que a maior parte dos médicos não possui tal formação.

Por ser uma terapia breve e centrada no diagnóstico, atribuindo ao sujeito um papel de doente, a sua eficácia em determinados quadros patológicos pode estar posta em causa, já que uma terapia breve pode nem sempre ser suficiente quando o quadro sintomático possui maior gravidade. Além disto, a atribuição do papel de doente ao sujeito pode nem sempre ser adequado, já que este pode não lidar bem com o seu estatuto de doente, recusando-se a aceitar tal aspecto. 
Dadas as evidências da sua eficácia, esta é uma terapia que deve ser mais difundida, necessitando de mais estudos que comprovem as suas potencialidades. Mesmo que não seja aplicada de forma exclusiva, as suas técnicas podem ser conjugadas com a aplicação de técnicas pertencentes a outras modalidades terapêuticas no mesmo processo terapêutico. Esta aborda áreas cruciais do desenvolvimento humano, bem como dificuldades frequentemente presentes nos sujeitos, pelo que a sua visão acerca dos relacionamentos humanos pode ser aproveitada a fim de obter melhorias na sintomatologia de diversas perturbações psiquiátricas.

Apesar de ter sido desenvolvida na década de 70, a terapia interpessoal permanece ainda desconhecida para muitos profissionais de saúde mental e dado o pouco conhecimento acerca desta modalidade psicoterapêutica, também a formação nesta área se encontra pouco difundida, sendo que muitos terapeutas apenas a aplicam com base no manual original, desconhecendo-se se tal facto será suficiente para uma correcta aplicação da mesma. Por este motivo, pode ser relativamente difícil para o sujeito conseguir submeter-se a esta terapia, já que não são muitos os técnicos com a devida formação e que pratiquem esta modalidade de intervenção.

Assim, a formação nesta área deve ser um aspecto a considerar por todos os terapeutas na área da saúde mental, ficando ao critério de cada um deles enveredar, ou não, pela intervenção terapêutica através desta modalidade de tratamento.

\section{REFERÊNCIAS}

ARCELUS, J. et al. A case series evaluation of a modified version of interpersonal psychotherapy (IPT) for the treatment of bulimic eating disorders: a pilot study. European Eating Disorders Review, v. 17, n. 4, p. 260-268, 2009.

BAHLS, S.; BAHLS, F. Psicoterapias da depressão na infância e na adolescência. Revista de Estudos de Psicologia, v. 20, n. 2, p. 25-34, 2003.

BAHLS, S.; ITO, M. Fundamentos da terapia interpessoal. Psico.utp.online, Curitiba, n. 3, p. 1-10, out. 2003.

BELLINO, S.; RINALDI, S.; BOGGETO, F. Adaptation of interpersonal psychotherapy to borderline personality disorder: a comparison of combined therapy and single pharmacotherapy. The Canadian Journal of Psychiatry, v. 55, n. 2, p. 74-82, 2010.

Campanini, R. et al. Efficacy of interpersonal therapy-group format adapted to post-traumatic stress disorder: an open-label add-on trial. Depression and anxiety, [S.1.], v. 27, n. 1, p. 72-77, 2010.

CURRY, J. Specific psychotherapies for childhood and adolescent depression. Biological Psychiatry, [S.1.], v. 49, n. 12, p. 1091-1100, 2001. 
FLECK, J. Terapia interpessoal: bases para a sua prática e resultados dos principais estudos. In: CORDIOLI, A. (Ed.). Psicoterapias: abordagens atuais. Porto Alegre: Artmed. 2008. v. 3, p. 204-222.

FRANK, E. et al. Interpersonal psychotherapy and antidepressant medication: evaluation of a sequential treatment strategy in women with recurrent maior depression. Journal of Clinical Psychiatry, [S.1.], v. 61, n. 1, p. 51-7, 2000.

KLERMAN, G. WEISSMAN, M. New applications of interpersonal psychotherapy. Washington: American Psychiatric Press, 1993.

LACAZ, F., BRESSAN, R., MELLO, M. A psicoterapia interpessoal na depressão em pacientes com esquizofrenia: proposta de um modelo terapêutico a partir de três casos clínicos. Revista de Psiquiatria, v. 27, n. 3, p. 252-261, 2005.

LEVENSON, H. et al. Concise guide to brief dynamic and interpersonal therapy. Washington: American Psychiatric Publisher. 2002.

MARKOWITZ, J. Interpersonal psychotherapy for dysthymic disorder. Washington: American Psychiatric Press. 1998

MARKOWITZ, J. Interpersonal psychotherapy. Washington: American Psychiatric Press.1998.

MARKOWITZ, J, SWARTZ, H. Case Formulation in Interpersonal Psychotherapy of depression. In: EELS, T. (Ed.). Handbook of psychotherapy case formulation. New York: Guilford, 2007. v. 2, p. 221-448.

MELLO, M. Terapia Interpessoal: um modelo breve e focal. Revista Brasileira de Psiquiatria, São Paulo, v. 26, n. 2, p. 124-130, 2004.

MELLO et al. A systematic review of research findings on the efficacy of interpersonal therapy for depressive disorders. European Archives of Psychiatry \& Clinical Neuroscience, [S.1.], v. 255, n. 2, p. 75-82, 2005.

MILLER, M. Using interpersonal therapy (IPT) with older adults today and tomorrow: a review of the literature and new developments. Current Psychiatry Reports, [S.1.], v. 10, n. 1, p. 16-22, 2008.

MUFSON, L. et al.. Interpersonal psychotherapy for depressed adolescents. New York: Guilford, 2004.

ROSSELÓ, J.; BERNAL, G. The efficacy of cognitive-behavioral and interpersonal treatments for depression in Puerto Rican adolescents. Journal of Consulting Clinical Psychology, [S.1.], v. 67, n. 5, p. 734-745, 1999. 
SCHESTATSKY, S.; FLECK, M. Psicoterapia das depressões. Revista Brasileira de Psiquiatria, São Paulo: v. 21, s. 1, p. 41-47. 1999.

SCHWARTZ, A. Interpersonal Therapy. In: LEHMANN, P. \& COADY, N. (Ed.). Theoretical perspectives for direct social work practice: a generalist-eclectic approach. New York: Springer, 2001. p. 128-144.

SWARTZ, H. et al. A Pilot Study of Brief Interpersonal Psychotherapy for Depression Among Women. Psychiatric Services, v. 55, n. 4, p. 448-450, 2004.

WEISSMAN, M. Mastering depression through interpersonal psychotherapy: Patient Workbook. New York: Oxford, 2005.

WEISSMAN, M.; MARKOWITZ, J.; KLERMAN, G. Clinician `s quick guide to interpersonal psychotherapy. New York: Oxford, 2007.

Recebido em: 15 de setembro de 2011 Aceito em: 19 de maio de 2012 